$\xi=$

\title{
Multidrug resistant Salmonella sp isolated from chicken
}

\author{
Atere*, Ayowole Victor \\ Department of Microbiology, Ekiti State University, P. M. B. 5363, Ado-Ekiti, Nigeria \\ *Corresponding author E-mail: victor_efosa001@yahoo.com
}

\begin{abstract}
Antibiotic resistance has been a major problem in combatting Salmonella in poultry, this research was designed to determine the antibiotic resistance level of Salmonella isolated from freshly dead chicken. A total of 107 freshly dead chicken were collected from 23 different farms, birds were necropsied, liver and trachea were collected, Salmonella were recovered from the samples using peptone water, Rappaport-Vassiliadis R10 Broth and Salmonella-Shigella agar. Pure culture were identified using cultural, morphological and biochemical characteristics. The pure isolates were subjected to antibiotic test using disc diffusion method. Sixteen isolates of Salmonella were recovered, 3 of which were from the trachea while 13 were recovered from the liver. All of the Salmonella isolates were resistant to Amoxicillin $100 \%$ while Nitrofuratoin was least resisted with only $37.5 \%$ of the Salmonella isolates showing resistance. The antibiotic resistant pattern often observed in this study were AMP, AUG, TLY with 75\% (12); AMP, AUG, TLY, CPR, ENR, 50\% (8); while 6.3\% (1) was resistant to all of the antibiotics tested. This result showed that there is an emergence of multi-resistance Salmonella in poultry, therefore it is important to carry out sensitivity test before administration of antibiotics in order to control poultry salmonellosis.
\end{abstract}

Keywords: Salmonella; Poultry Feed; Resistance; Salmonellosis.

\section{Introduction}

In animal production systems, antibiotics are used for both therapeutic and non-therapeutic purposes. Non-therapeutically, antibiotics are used as growth promoters in livestock and poultry (Johnson, 2001; McEwen and Fedorka-Cray, 2002). Antibiotics are also used to improve the general hygiene in barns. This non-therapeutic use of antibiotics in feed may lead to increased levels of antibiotic resistance in both the pathogens and fecal micro flora of poultry (Witte 2000; Van-Veen et al., 2001).

The development of resistance in poultry pathogens may undermine the efficacy and utility of antibiotics used to control these infections. Infections of domestic poultry with Salmonella are expensive both for the poultry industry and for society as a whole (Amand et al., 2013). Some Salmonella serovars can affect multiple host species and it makes a serious problem according to the food chain (Jung et al., 2011). There is evidence that eggs and poultry meat are two of the most important sources of Salmonella associated with human infection (Arsenault et al., 2007).

Salmonella species isolated from clinical and environmental sources has shown an increased resistance to antibiotics since it has developed a number of elaborate mechanisms for acquiring and disseminating plasmids, transposons, phages, and other genetic determinants (Harts and Kaariuki, 1998). Therefore, this study focuses on the resistance of Salmonella species isolated from freshly dead chicken.

\section{Materials and methods}

\subsection{Sample collection}

One hundred and seven (107) freshly dead chicken were collected from 23 different farms in Ondo and Ekiti State (South Western,
Nigeria) between January and June 2015. The samples were conveyed to the laboratory for analysis within 2 hours of collection.

\subsection{Bacteriology}

The Freshly dead chicken were necropsied, the liver and trachea were collected. Swabs were collected aseptically from the trachea and the liver for bacteria isolation. The swabs collected was activated in buffered peptone water for 1 hour at $37^{\circ} \mathrm{C}$. A loop full of the activated organisms in the buffered peptone water was inoculated into a selective enrichment medium Rappaport-Vassiliadis R10 Broth. Thereafter, a loop full of the activated organism in the Rappaport-Vassiliadis broth was inoculated on SalmonellaShigella agar and nutrient agar (Biomark) and incubated for 24 hours at $37^{\circ} \mathrm{C}$ temperature in an incubator (Royalcare England. DNP 9022A) (Ramya et al., 2012). Further confirmation of Salmonella was done by Gram reaction, motility, catalase, oxidase, nitrate, urease, indole, methyl red, Voges-Proskauer, and citrate tests (Atere et al., 2015a).

\subsection{Antibiotic Susceptibility test}

In vitro susceptibility of the identified Salmonella isolates against antimicrobial agents was determined by the standard disk diffusion procedure. The organisms were standardized using McFarland standard at the absorbance of $450 \mathrm{~nm}$. The samples were inoculated on Muller-Hinton agar. The following antimicrobial agents were tested: Ceftazidime (CAZ 30 $\mu \mathrm{g}$ ), Cefuroxime (CRX

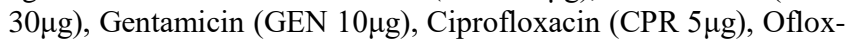

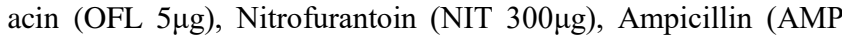

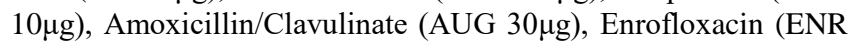

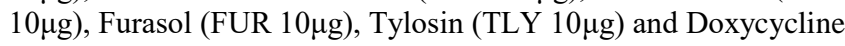

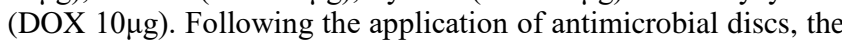
plates were incubated at $37{ }^{\circ} \mathrm{C}$ for $24 \mathrm{~h}$ in an incubator (Royalcare England. DNP 9022A). The diameters of the zones of inhibition 
were measured (millimetres) and were compared to internationally accepted standard to determine the susceptibility or resistance of the isolate (Atere et al., 2015a).

\section{Results}

Salmonella was recovered from 12 of the 23 farms. A total of 16 Salmonella isolates were recovered, 3 of the isolates was isolated from the trachea while 13 were from the liver of the birds. The cultural characteristics of the isolates were small round pale convex colony on nutrient agar and black spot colony on SalmonellaShigella agar. They are gram negative rods, catalase positive, methylred positive and are motile. The isolates were negative to indole, citrate, oxidase, urease and voges-proskauer.

The antibiogram of the Salmonella isolates showed that all showed $100 \%$ resistance to Amoxicillin while Nitrofuratoin was least resisted with only $37.5 \%$ of the Salmonella isolates showing resistance (Table 1). All the isolates of Salmonella recovered in this study were resistant to at least four antibiotic. Only one of the isolates showed resistance to only 4 antibiotics while all others were resistant to between 5 and 12 antibiotics. One of the Salmonella isolates showed resistance to all the antibiotics used in this research (Table 2). The pattern of antibiotic resistance showed that $75 \%$ of the isolates had resistance pattern AMP, AUG, TLY while $50 \%$ had AMP, AUG, TLY, CPR, ENR pattern.

Table 1: Percentage (\%) Antibiotic Resistant of Salmonella Sp. Isolated from Chicken

\begin{tabular}{|c|c|c|c|c|c|c|c|c|c|c|c|c|}
\hline Isolates & AMP & AUG & OFL & TLY & CPR & ENR & DOX & FUR & GEN & NIT & CAZ & CRX \\
\hline Trachea & 100 & 100 & 33.3 & 100 & 100 & 33.3 & 66.7 & 66.7 & 33.3 & 33.3 & 100 & 66.7 \\
\hline$(\mathrm{n}=3)$ & (3) & (3) & (1) & (3) & (3) & (1) & (2) & (2) & (1) & (1) & (3) & (2) \\
\hline Liver & 92.3 & 100 & 76.9 & 76.9 & 61.5 & 92.3 & 92.3 & 61.5 & 84.6 & 38.5 & 53.8 & 53.8 \\
\hline$(\mathrm{n}=13)$ & (12) & (13) & (10) & (10) & (8) & (12) & (12) & (8) & (11) & (5) & (7) & (7) \\
\hline Total & 93.8 & 100 & 68.8 & 81.2 & 68.8 & 81.2 & 87.5 & 62.5 & 75.0 & 37.5 & 62.5 & 56.3 \\
\hline $\mathrm{n}=16$ & (15) & (16) & (11) & (13) & (11) & (13) & (14) & (10) & (12) & (6) & (10) & (9) \\
\hline
\end{tabular}

Key: Ampicillin (AMP), Amoxicillin/Clavulinate (AUG), Ofloxacin (OFL), Tylosin (TLY), Ciprofloxacin (CPR), Enrofloxacin (ENR), Doxycycline

(DOX), Furasol (FUR), Gentamicin (GEN), Nitrofurantoin (NIT), Ceftazidime (CAZ) and Cefuroxime (CRX).

Table 2: Pattern of Antibiotic Resistance in Salmonella Isolates

\begin{tabular}{lll}
\hline Antibiotics & No of Isolates \\
\hline AMP, AUG, TLY & 12 & Percentage \% \\
CAZ, CRX, GEN & 6 & 75.0 \\
NIT, OFL, GEN & 4 & 37.5 \\
DOX, FUR, ENR, TLY & 5 & 25.0 \\
AMP, AUG, TLY,CPR, ENR & 8 & 31.3 \\
FUR, ENR, CPR,TLY & 4 & 50.0 \\
GEN, CRX, NIT, CAZ & 3 & 25.0 \\
AMP, AUG, OFL, TLY, CPR, ENR,NIT,GEN, CRX, DOX, CAZ, FUR & 1 & 18.8 \\
\hline
\end{tabular}

Key: Ampicillin (AMP), Amoxicillin/Clavulinate (AUG), Ofloxacin (OFL), Tylosin (TLY), Ciprofloxacin (CPR), Enrofloxacin (ENR), Doxycycline (DOX), Furasol (FUR), Gentamicin (GEN), Nitrofurantoin (NIT), Ceftazidime (CAZ) and Cefuroxime (CRX).

\section{Discussion}

Salmonellosis is an infectious disease of humans and animals caused by Salmonella sp a facultative intracellular pathogen causing localized or systemic infections, in addition to a chronic asymptomatic carrier state. Infection of Salmonella in poultry is often referred to as fowl typhoid, which affect birds of all ages (Ramya et al., 2012). Salmonella is most found in the gastro intestinal tract of birds, however, because of their invasive mechanism, they get their way into tissues of the host. In infected birds, the liver serves as the resting place for the organism (Johnston, 2001). Since Salmonella infection could be systemic, isolating Salmonel$l a$ from the trachea of the birds is possible. In previous reports Salmonella had been isolated from the tissue, lungs, liver gastrointestinal tract of the infected birds (Hernandez et al., 2005).

Salmonella in poultry is traceable to the poultry feeds and water, as well as the hygiene of the poultry (environmental factor) and sometimes from humans (Roy et al., 2006). Atere et al. (2015a) reported isolating Salmonella from the poultry feeds, this is an indication that the type of feed or the hygiene of the feeds can have a great effect on the microbial contamination of the poultry which can later transfer an effect of economic importance on the farmer.

The antibiotic susceptibility of the isolates of Salmonella in this research showed multiple resistance where they resist at least four antibiotics. In other research where samples are collected from poultry, the resistance is not as high as observed in this study. Nchawa and Bassey (2015) reported the antibiotic susceptibility of Salmonella isolates in poultry where it was found that $72.9 \%$ resisted AMP, AUG, 29.4\% and CAZ 19.5\%, this result is lower to what was observed for this study. Oluyege and Oyinloye (2013) also reported the antibiotic resistance of Salmonella isolated from poultry as AUG $73.5 \%$, OFL $20.9 \%$ and CPR $35.3 \%$ which is also lower compare to what was observed in this research. The high level of resistance observed in this research may have been as a result of the type of sample collected and the effect of misuse of antibiotics by the farmer. Another possible reason for the multiple resistance of Salmonella could be attributed to the proliferation of fake or sub-standard drug in Nigeria (Dashe et al., 2013). The high sensitivity of the isolated organisms to Nitrofurantoin, could be related to less frequent usage of this drug for therapeutic purposes, therefore reducing the chance of resistance to develop (Moham$\operatorname{mad}$ et al., 2012).

The major factors responsible for antimicrobial resistance in bacteria is misuse of antibiotic, crowding and poor sanitation. These three factors are typical of intensive poultry farming and explain the high prevalence and degree of resistance in Salmonella of poultry origin (Van-den Bogaard,et al., 2000). The source of the resistance may also have come from the poultry feeds consumed, since antibiotics are used as feed additives to improve feed efficiency and weight gain (Tabatabaeci and Nasirian, 2003; Helmuth and Hensel, 2004). Many antibiotics are also used in feed and water to control disease. Indiscriminate use of antibiotics has provided selective pressure for the emergence of drug resistant strains of bacteria associated with poultry products. In a research carried out by Atere et al. (2015b), a similar report was given on the antibiotic susceptibility of Escherichia coli which is far higher in poultry (freshly dead chicken) than in the samples isolated from environment. The reason for the high level of antibiotic resistance was attributed to misuse of antibiotics before clinical reports. In conclusion, there is a need to create more awareness among the poultry farmers in Nigerian about the proper use of antibiotics, importance of clinical and laboratory test before administration of antibiotics, proper hygiene and effect the type of feed adopted can have on their birds. Since Salmonella is zoonotic, it is of public health importance because these resistance gene can easily be transferred to human population. 


\section{Acknowledgements}

The Author appreciate the assistance received from Dr. O. S Alo, Dr. O. A. Ajurojo and Mr. O. J. Bello of Metrovet Veterinary Hospital Ado-Ekiti, Ekiti State for their assistance during collection of samples.

\section{References}

[1] Amand, J. A., Otto, S. J., Cassis, R., Annett-Christianson, C. (2013). Antimicrobial resistance of Salmonella enteric serovar Heidelberg isolated from poultry in Alberta. Avian Pathology. 42(4): 379-386. http://dx.doi.org/10.1080/03079457.2013.811465.

[2] Arsenault J, Letellier A, Quessy S, Morin J, Boulianne M. (2007). Prevalence and Risk Factors for Salmonella and Campylobacter spp. Carcass Contamination in Turkeys Slaughtered in Quebec, Canada. Journal of Food Protection. 70 (6): 1350-1359.

[3] Atere, V. A, Bamikole, A. M. and Ajurojo, O. A. (2015a). Antibiotic Susceptibility of Bacteria Isolated from Poultry Feeds Sold in Ado Ekiti, Nigeria. Journal of Advancement in Medical and Life Sciences V3I2. DOI: 10.15297/JALS.V3I2.06.

[4] Atere, V. A., Bamikole, A. M., Ajurojo, O. A. and Alo, O. S (2015b). Antimicrobial Resistance Pattern of Pathogenic Escherichia coli Isolated from Chicken Liver and Trachea. Journal of Advancement in Medical and Life Sciences. V3I3. DOI: 10.15297/JALS.V3I3.02

[5] Dashe, Y. D., Raji, M. A., Abdu, P. A., Oladele, B. S. and Sugun, M. Y. (2013). Multidrug Resistant Pasteurella multocida Strains Isolated from Chickens with Cases of Fowl Cholera in Jos, Nigeria. Int. J. Poult. Sci., $12 \quad$ (10): $596-600$ http://dx.doi.org/10.3923/ijps.2013.596.600.

[6] Harts CA, Kariuki S. (1998) Antimicrobial resistance in developing countries. Biomedical Journal, 37:647-650.

[7] Helmuth, R. and Hensel, A. (2004). Towards the rational use of antibiotics: results of the first international symposium on the risk analysis of antibiotic resistance. J. Vet. Med. 51: 357-360. http://dx.doi.org/10.1111/j.1439-0450.2004.00778.x.

[8] Hernandez, T., Sierra, A., Rodrigue-Alvarez, C., Torres, A., Arevalo, M.P., Calvo, M. and Arias, A (2005). Salmonella enteric serotypes isolated from imported frozen chicken meat in the Canary Islands. J Food Prot., 68(12):2702-2706.

[9] Johnston, A. M. (2001). Animals and antibiotics. Int. J. Antimicrob. Agents. 18:291-294. http://dx.doi.org/10.1016/S09248579(01)00379-X.

[10] Jung, H., Lee, S., Kim, Ch., Sunwoo, S., Lyoo, Y. S. (2011) Serovars distribution and antimicrobial resistance patterns of Salmonella spp. isolated from the swine farms and slaughter houses. Korean J. Vet. Res. 51(2): 123-128.

[11] McEwen, S. A. and Fedorka-Cray, P. J. (2002). Antimicrobial use and resistance in animals. Clin. Infect. Dis. 34:93-106. http://dx.doi.org/10.1086/340246.

[12] Mohammad, J., Ahmad, R. and Mohsen, N. (2012). A study on bacterial flora and antibacterial resistance of yolk sac infection in Japanese quail (Coturnix japonica). Comp. Clin. Pathol. 80: (12) 1459 69.

[13] Nchawa, Y. Y and Bassey, E. B (2015), Antimicrobial Susceptibility Patterns of Salmonella Species from Sources in Poultry Production Settings in Calabar, Cross River State, Nigeria. American Journal of Health Research 3(2): 76-81. http://dx.doi.org/10.11648/j.ajhr.20150302.14.

[14] Oluyege, J.O and .Oyinloye, I.A. (2013) Incidence of multiple antibiotic resistance among Salmonella spp. isolated from poultry droppings and cow dung in Ado-Ekiti metropolis Researcher; 5(9) 32-36. http://www.sciencepub.net/researcher

[15] Ramya, P., Madhavarao, T. and Rao, L. V. (2012). Study on the incidence of Salmonella enteritidis in poultry and meat samples by cultural and PCR methods. Vet World, 5(9): 541-54. http://dx.doi.org/10.5455/vetworld.2012.541-545.

[16] Roy, P., Purushothaman, V., Koteeswaran, A. and. Dhillon, A. S. (2006). Isolation, Characterization and Antimicrobial Drug Resistance Pattern of Escherichia coli Isolated from Japanese quail and their Environment. J. Appl. Poult. Res. 15:442-446. http://dx.doi.org/10.1093/japr/15.3.442.

[17] Tabatabaeci, R. R. and Nasirian, A. (2003). Isolation, identification and antimicrobial resistance patterns of $E$. coli isolated from chicken flocks. Iranian J. Pharm. Therafoenlizion. 2:39-42.

[18] Van-den Bogaard, A. E., London, N. and Stobberingh, E. E. (2000) Antimicrobial resistance in pig faecal samples from The Nether- lands (five abattoirs) and Sweden. J. Antimicrob. Chemother. 45: 663-671. http://dx.doi.org/10.1093/jac/45.5.663.

[19] Witte, W. (2000). Selective pressure by antibiotic use in livestock. Int. J. Antimicrob. Agents. 16:S19-S24 http://dx.doi.org/10.1016/S0924-8579(00)00301-0. 